\title{
Reliability of a method to conduct upper airway analysis in cone-beam computed tomography
}

Karen Regina Siqueira de Souza Paula Vanessa Pedron OltramariNavarro

Ricardo de Lima Navarro

Ana Cláudia de Castro Ferreira Conti

Marcio Rodrigues de Almeida

Department of Orthodontics, School of Dentistry, Univ Norte do Paraná - Unopar, Londrina, PR, Brazil.
Declaration of Interests: The authors certify that they have no commercial or associative interest that represents a conflict of interest in connection with the manuscript.

\section{Corresponding Author:}

Paula Vanessa Pedron Oltramari-Navarro

E-mail:pvoltramari@hotmail.com

Submitted: Sep 09, 2012

Accepted for publication: Nov 22, 2012

Last revision: Dec 06, 2012

\begin{abstract}
The aim of this study was to assess the reliability of a method to measure the following upper airway dimensions: total volume (TV), the nasopharyngeal narrowest areas (NNA), and the oropharyngeal narrowest areas (ONA). The sample consisted of 60 cone-beam computed tomography (CBCT) scans, evaluated by two observers twice, using the Dolphin 3D software (Dolphin Imaging \& Management solutions, Chatsworth, California, USA), which afforded image reconstruction, and measurement of the aforementioned dimensions. The data was submitted to reliability tests, by the intraclass correlation coefficient (ICC), and the Bland \& Altman agreement tests, with their respective confidence intervals $(\mathrm{CI})$ set at $95 \%$. Excellent intra- and interobserver reliability values were found for all variables assessed (TV, NNA and ONA), with ICC values ranging from 0.88 to 0.99 . The data demonstrated an agreement between the two assessments of each observer and between the first evaluations of both observers, thus confirming the reliability of this methodology. The results suggest that this methodology can be used in further studies to investigate upper airway dimensions (TV, NNA, and ONA), thereby contributing to the diagnosis of upper airway obstructions.
\end{abstract}

Descriptors: Orthodontics; Diagnosis; Tomography; Respiration.

\section{Introduction}

The effects of breathing and its participation in craniofacial growth and development have been the object of important research concerning orthodontic diagnoses and treatment plans. Countless studies point out that breathing through upper airways is of great importance for normal craniofacial development. ${ }^{1-6}$ Alterations in upper airway breathing, particularly during facial growth, may affect the development of structures and functions of the stomatognathic system. ${ }^{7}$

A lateral cephalogram is part of early orthodontic documentation, and its assessment has allowed orthodontists to view upper airway obstructions. ${ }^{1-3}$ However, measurements in the sagittal plane are not accurate, insofar as this methodology has important limitations, with errors inherent to a two-dimensional representation of complex tridimensional structures. ${ }^{1,4,5}$

Hence, cone-beam computed tomography (CBCT) has been introduced as a new and effective diagnosis method to evaluate upper airways, considering that images are obtained in three dimensions (3D). ${ }^{1,6,8}$ 


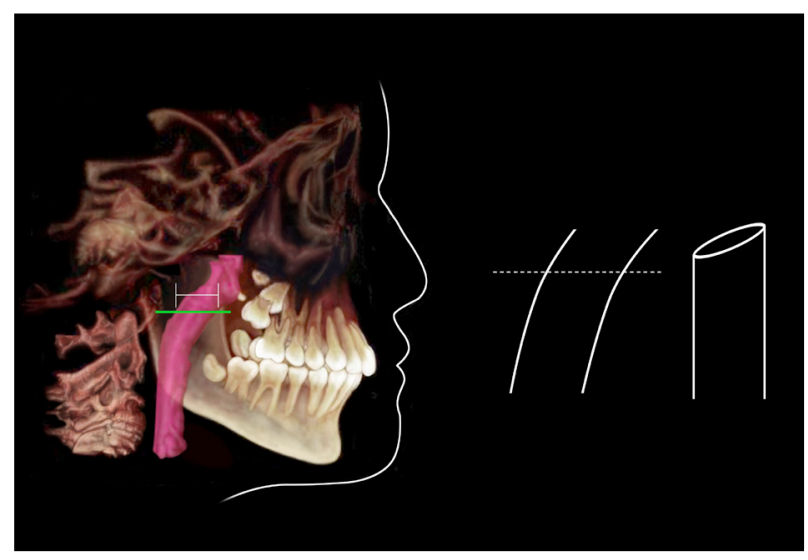

Figure 1 - Assessment of NNA without vertical correction.

Despite the excellent reliability of CBCT scans, a great diversity of methodologies to evaluate upper airways persists to date. ${ }^{1,8,9}$

According to some studies, ${ }^{1,10,11}$ the use of reliable methods in clinical practice is essential, since observational and visual evaluations are subjective, and may alter the results of programs that involve intervention. Thus, should a non-reliable method be used, both the diagnosis and the treatment plan may suffer alterations.

Although a number of studies have proven reliable in their specific methodologies for conducting a tridimensional evaluation of the upper airways (including total volume and minimum axial areas), ${ }^{1,5,11-13}$ there is no consensus in the existing literature as to the ideal method, owing especially to the complex anatomy of this region. ${ }^{1,8}$ Moreover, most of the programs in use have limitations in performing measurements in curvilinear regions, especially in the nasopharyngeal region (Figures 1 and 2). These limitations may jeopardize proper evaluation of the minimum axial area. Additionally, smaller areas may lead to airway obstructions or limit the airflow, and our interest is to find the greatest constrictions perpendicular to the airway, and, consequently, to the airflow. The nasopharyngeal minimum perpendicular area, however, is not easy to find, insofar as the software tool locates and calculates the minimum area parallel to the axial plane, instead of the minimum area perpendicular to the airway.

This study, therefore, aimed at evaluating the re-

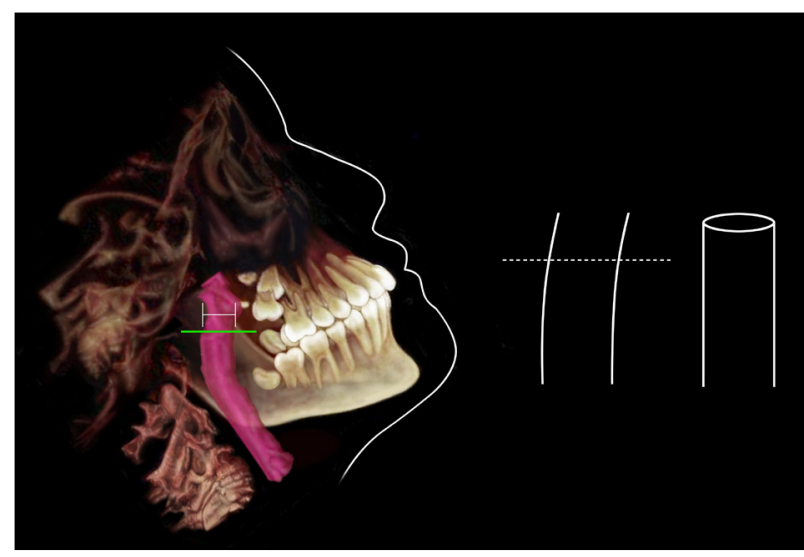

Figure 2 - Correction of the posterior walls of NNA, to render them vertical for analysis, and to afford an actual assessment of these areas in curvilinear regions.

liability of a method used to measure upper airway dimensions, including total volume (TV), the nasopharyngeal narrowest areas (NNA), and the oropharyngeal narrowest areas (ONA).

\section{Methodology}

The protocol of this study was approved by the Research Ethics Committee of the University of North Parana.

Sixty patients with a mean age of 17.86 years were selected for this study from the records of an oral radiology clinic. The first inclusion criterion was that every patient needed to take a 12 " CBCT scan showing all the craniofacial structures required for the analysis. Moreover, patients could not have been submitted to an orthognathic surgery, or have been diagnosed with a syndrome, or a detectable pathology along the upper airways, observable by inspection of the images.

All CBCT scans were carried out by a single experienced radiologist, using the same tomographer (i-Cat Imaging Sciences International, Hatfield, USA); the scanning protocol was $120 \mathrm{kV}, 36.9 \mathrm{~mA}$, $13 \times 23 \mathrm{~cm}$ field of view, $0.4-\mathrm{mm}$ voxel, and scanning time of 40 seconds. Before conducting the CBCT, patients were asked not to swallow, nor to move their heads during the exam. ${ }^{5,12}$ Moreover, the CBCT had to be taken with the patient in occlusion to reduce the variations of the mandibular position and of soft-tissue airway measurements, often asso- 


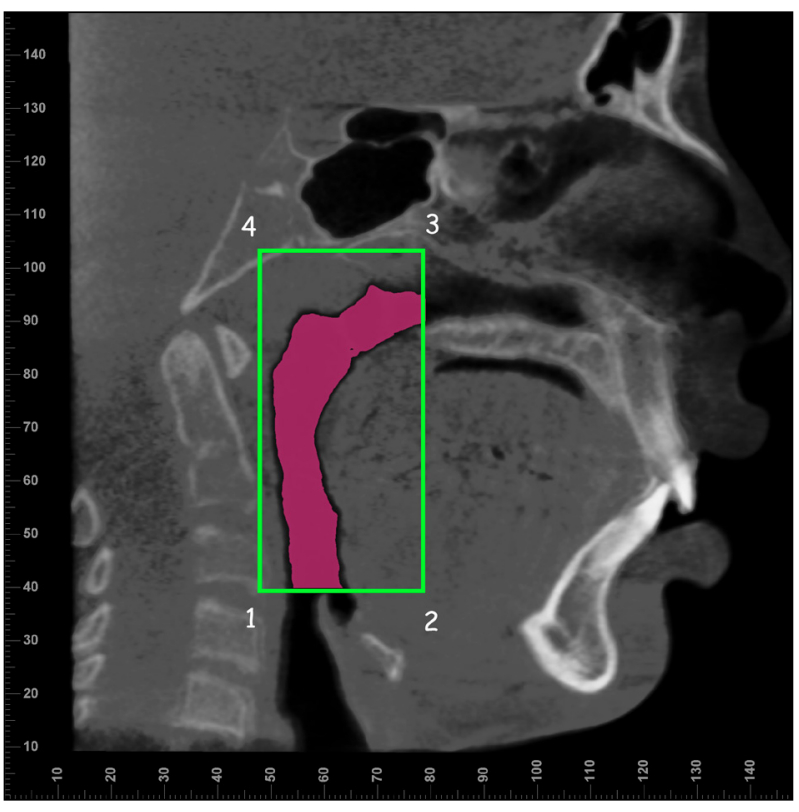

Figure 3 - Determination of anatomical limits in sagittal and coronal planes for the total volume (TV) of upper airways.

ciated with the resting position. ${ }^{14}$

Two observers evaluated all the $60 \mathrm{CBCT}$ scans twice, regarding TV, NNA ONA, with the Dolphin 3D program (version $11.5^{\circledR}$, Dolphin Imaging \& Management Solutions, Chatsworth, USA). Before starting the analysis, each patient's head position was corrected with the Dolphin software tools, using the midsagittal, the Frankfort horizontal and the transporionic ${ }^{15,16}$ planes as references, considering that small deviations in the position of the head are bound to exist.

\section{Upper airway total volume (TV) assessment}

Delimitation of the upper airway TV (nasopharynx and oropharynx) was conducted by an initial analysis of a sagittal plane image reconstruction. The following anatomic limits were then determined sequentially:

1. Lower limit: after locating the caudal medial point of the third cervical vertebra, a straight line was drawn towards the anterior limit, until the projection of the posterior nasal spine (PNS) could be seen; ${ }^{12}$

2. Anterior limit: a straight vertical line passing through the posterior nasal spine (PNS) point;

3. Upper limit: a horizontal line passing through the lower point on the border of the sphenoidal sinus;

4. Posterior limit: a vertical plane was determined from the upper limit up to the caudal medial point of the third cervical vertebra, until the anatomic delimitation was completed in the sagittal plane.

In addition, the limits were checked in the coronal and axial planes, to certify that the lateral wall of the pharynx included all lateral projections. After conducting the anatomic delimitation, the entire aerial space was outlined, and filled in with seed points. Another check had to be complete in all visualization planes, to make sure that the area outlined with seed points had been totally filled in (Figure 3).

\section{Upper airway nasopharyngeal narrowest area (NNA) and oropharyngeal narrowest area (ONA) assessment}

The same anatomic limits that were determined to calculate TV were also used to assess NNA and ONA. However, due to the curvilinear anatomy of this region, the correction of the posterior walls of NNA (Figures 1 and 2) and of ONA was performed manually and separately, in order to make them vertical during the analysis, and to afford a true assessment of these areas. Thus, for each segmented patch, the program determined the narrowest area to be quantified in square millimeters $\left(\mathrm{mm}^{2}\right)$ (Figures 4 and 5).

Thirty days after the first evaluation, all measurements were remade by both examiners, and the results submitted to the intraclass correlation coefficient (ICC) $)^{17}$ and the Bland \& Altman agreement tests, with their respective confidence intervals set at $95 \%$. These tests enabled the evaluation of intraand interobserver reliability values for all assessed variables (TV, NNA and ONA), following the criteria described by Fleiss. ${ }^{18}$ The first measurements of each observer were used to calculate interobserver reliability. Data were analyzed using SPSS version 10 (Chicago, IL, USA) and MedCalc version 8.1.0.0 (Mariakerke, Belgium) software. 


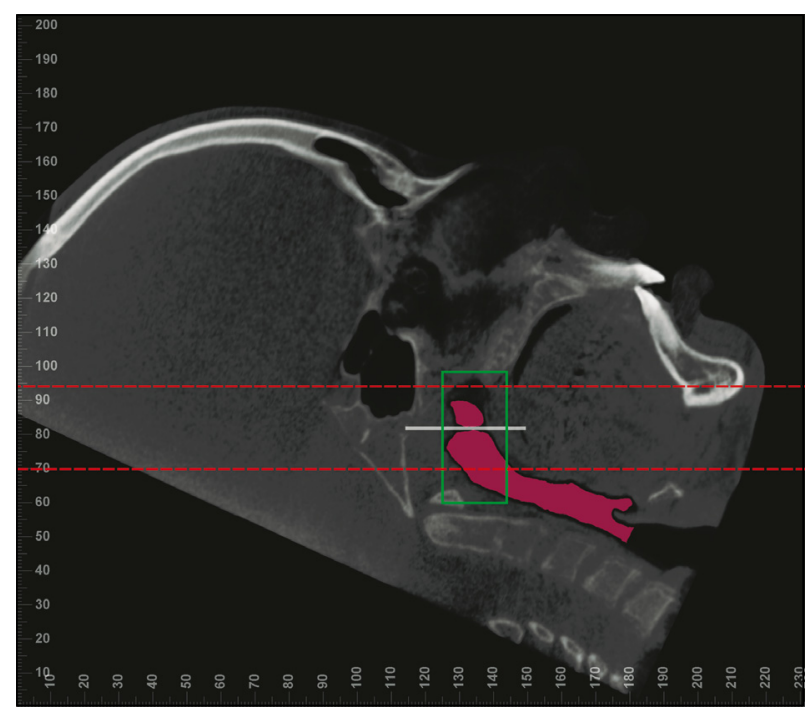

Figure 4 - Assessment of upper airway nasopharyngeal narrowest area.

\section{Results}

Excellent reliability ${ }^{18}$ was observed for all assessed variables (VT, NNA and ONA). Regarding the intraexaminer errors of Researcher 1, high reliability was observed for all variables (ICC ranging from 0.98 to 0.99 ), with very narrow confidence intervals, thereby showing excellent agreement for these measurements (Table 1). Similar results were observed for the intraexaminer errors of Researcher 2 , with ICC values ranging from 0.93 to 0.99 (Table 2). The interexaminer evaluation also showed high reliability, with ICC ranging from 0.88 to 0.98 (Table 3).

Agreement was thus asserted between the examiners in the two evaluations, and reproducibility of this methodology was confirmed.

\section{Discussion}

Regarding image diagnosis, a lateral cephalogram is part of early orthodontic documentation, and its assessment has allowed orthodontists to observe possible upper airway obstructions. However, measurements in the sagittal plane are not accurate, insofar as the methodology used has important limitations, with errors inherent to a two-dimensional representation of complex three-dimensional structures. Cone-beam computed tomography (CBCT) has been introduced as a new and effective diagnosis

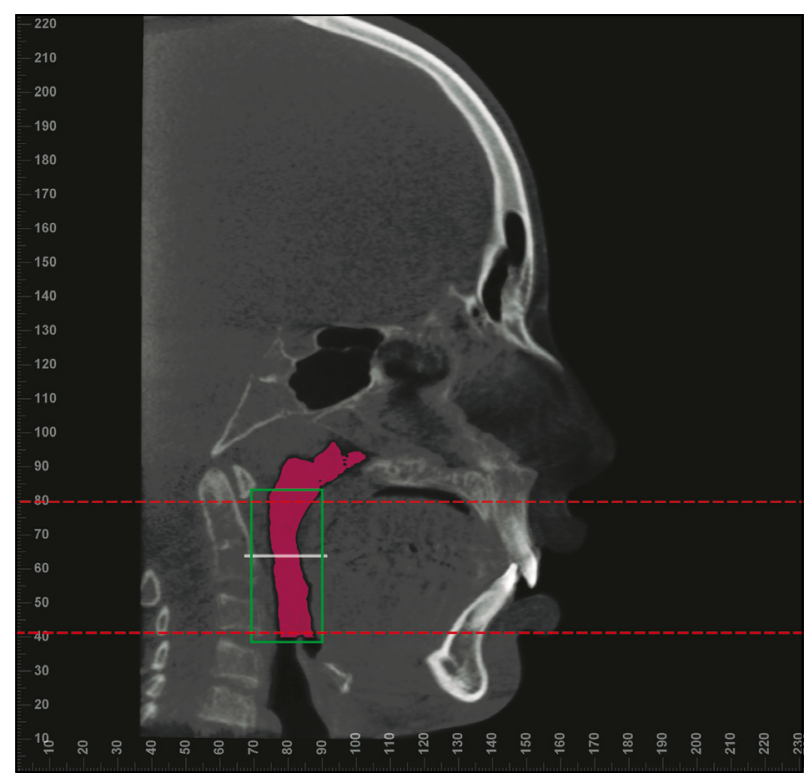

Figure $\mathbf{5}$ - Assessment of upper airway oropharyngeal narrowest area.

method to evaluate upper airways (UAW), considering that images are obtained in three dimensions (3D). This permits the real evaluation of total volume (TV) and of minimum axial areas, thus contributing to improving the diagnosis. ${ }^{1,5,7,8,12}$

In fact, the advent of CBCT has afforded highly reliable tests that, in turn, have led to remarkable developments in dentistry, regarding diagnosis and treatment management. ${ }^{1,4,19}$ Nevertheless, despite the high reliability of this exam, a great variety of methodologies have been proposed to analyze these images, mainly of upper airway areas. Thus, the aim of this study was to describe a methodology based on anatomic repairs that could be easily identified and measured, and that could be reproducible in other studies, by means of a specific statistical analysis for this purpose..$^{10,17}$

In this study, excellent intraobserver reliability was found for both examiners, for all variables assessed (VT, NNA and ONA), with ICC values ranging from 0.93 to 0.99 (Tables 1 and 2). Interobserver reliability was also excellent, but with values ranging from 0.88 to 0.99 (Table 3). These results demonstrated that intraexaminer reliability is usually better than interexaminer reliability. This suggests a greater likelihood of achieving uniformity for the same observer than among different examiners. ${ }^{20,21}$ 
Table 1 - Intraexaminer errors (Researcher 1) for total volume (TV), nasopharyngeal narrowest area (NNA) and oropharyngeal narrowest area (ONA): mean (M), standard deviation (SD), Bland \& Altman agreement and intraclass correlation coefficient (ICC).

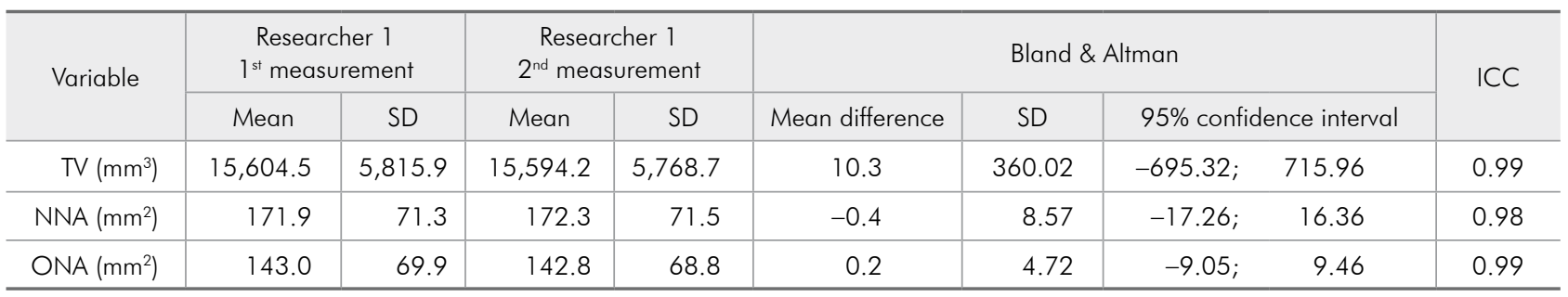

Table 2 - Intraexaminer errors (Researcher 2) for total volume (TV), nasopharyngeal narrowest area (NNA) and oropharyngeal narrowest area $(\mathrm{ONA})$ : mean $(\mathrm{M})$, standard deviation (SD), Bland \& Altman agreement and intraclass correlation coefficient (ICC).

\begin{tabular}{|c|c|c|c|c|c|c|c|c|c|}
\hline \multirow{2}{*}{ Variable } & \multicolumn{2}{|c|}{$\begin{array}{l}\text { Researcher } 2 \\
1^{\text {st }} \text { measurement }\end{array}$} & \multicolumn{2}{|c|}{$\begin{array}{c}\text { Researcher } 2 \\
2^{\text {nd }} \text { measurement }\end{array}$} & \multicolumn{4}{|c|}{ Bland \& Altman } & \multirow{2}{*}{ ICC } \\
\hline & Mean & SD & Mean & SD & Mean difference & SD & $95 \%$ confic & e interval & \\
\hline $\mathrm{TV}\left(\mathrm{mm}^{3}\right)$ & $15,910.8$ & $6,091.8$ & $15,836.5$ & $5,998.1$ & 74.3 & 637.57 & $-1,175.36$ & 1323.94 & 0.99 \\
\hline $\mathrm{NNA}\left(\mathrm{mm}^{2}\right)$ & 162.5 & 76.4 & 161.3 & 70.1 & 1.2 & 31.40 & -60.36 & 62.75 & 0.93 \\
\hline ONA $\left(\mathrm{mm}^{2}\right)$ & 141.9 & 71.1 & 142.9 & 71.0 & -1.0 & 10.41 & -21.41 ; & 19.41 & 0.98 \\
\hline
\end{tabular}

Table 3 - Interexaminer errors (Researcher 1 vs. Researcher 2) for total volume (TV), nasopharyngeal narrowest area (NNA) and oropharyngeal narrowest area (ONA): mean (M), standard deviation (SD), Bland \& Altman agreement and intraclass correlation coefficient (ICC).

\begin{tabular}{|c|c|c|c|c|c|c|c|c|c|}
\hline \multirow{2}{*}{ Variable } & \multicolumn{2}{|c|}{$\begin{array}{l}\text { Researcher } 1 \\
1^{\text {st }} \text { Measurement }\end{array}$} & \multicolumn{2}{|c|}{$\begin{array}{c}\text { Researcher } 2 \\
1^{\text {st }} \text { measurement }\end{array}$} & \multicolumn{4}{|c|}{ Bland \& Altman } & \multirow[t]{2}{*}{ ICC } \\
\hline & Mean & SD & Mean & SD & Mean difference & SD & $95 \%$ confid & ce interval & \\
\hline $\mathrm{TV}\left(\mathrm{mm}^{3}\right)$ & $15,604.5$ & $5,815.9$ & $15,910.8$ & $6,091.8$ & -306.28 & 1573.3 & $-3,390.36$; & $2,777.53$ & 0.95 \\
\hline $\mathrm{NNA}\left(\mathrm{mm}^{2}\right)$ & 171.9 & 71.3 & 162.5 & 76.4 & 9.38 & 29.87 & $-49.17 ;$ & 67.94 & 0.88 \\
\hline ONA $\left(\mathrm{mm}^{2}\right)$ & 143.0 & 69.9 & 141.9 & 71.1 & 1.09 & 14.59 & -27.49 ; & 29.69 & 0.98 \\
\hline
\end{tabular}

Additionally, the variation of all measurements ranged within acceptable limits, showing that two evaluations for each measurement (TV, NNA, and ONA), carried out by two examiners experienced in handling the Dolphin ${ }^{\circledR}$ program, tend to produce similar results. Therefore, this methodology proved highly reliable (Table 3 ).

The lowest ICC value was found for NNA (Tables 1-3), for both intra- and interexaminer measurements. This result can be attributed to the great topographic variability at the nasopharynx level, due to the complex anatomy of this region. ${ }^{11}$ This study sought to minimize this limitation by dividing the upper airways into nasopharynx and oro- pharynx for evaluation purposes, thereby affording a more reliable NNA delimitation. ${ }^{1,5,11,13}$ Thus, the correction of the posterior NNA and ONA walls was performed separately, so as to make them vertical for analysis, and to afford a true assessment of these areas in curvilinear regions, as shown in Figures 1 and 2 .

In addition, it is important to point out that if examiners not experienced in the anatomy of the region carry out this methodology, the execution of the proposed method may be jeopardized. This rationale has already been reported in the literature, and is probably a limiting factor to obtaining the standard mean values that could be used as deter- 
mining factors in the diagnosis. ${ }^{1,5,8}$

A reliability analysis requires two statistical tests -in this case that of ICC and that of Bland \& Altman-because an ICC test alone does not provide sufficient information about the reliability of the measures. This is because the values measured and variations thereof, as well as measurement errors, are not shown, and also because it is impossible to make a clinical interpretation. The Bland and Alt$\operatorname{man}^{10}$ results, used to interpret the size and scope of measurement differences more easily, complement the correlation analysis by examining the different patterns between both measurements, i.e., test measure variations in relation to the mean difference. ${ }^{11,22}$ In addition, this method shows confidence interval values for the mean difference, and for agreement limits. This data, will indicate the measurement errors, and may relate to clinical acceptance. ${ }^{22}$ Thus, the tests used in this study are appropriate to assess the reliability of the method proposed in cases of similar designs. Furthermore, the ICC test is currently regarded as important to evaluate intraexaminer correlation, insofar as it allows correlation analysis, and agreement among results. ${ }^{22}$

It may therefore be concluded that the tridimensional evaluation of TV, NNA, and ONA made by actually picturing the morphology of this area is fundamental in clinical practice, since pathologies such as mouth breathing and obstructive sleep apnea are frequent, although not always related to areas of narrowing of upper airways. Accordingly,

\section{References}

1. Aboudara C, Nielsen I, Huang JC, Maki K, Miller AJ, Hatcher D. Comparison of airway space with conventional lateral headfilms and 3-dimensional reconstruction from cone-beam computed tomography. Am J Orthod Dentofacial Orthop. 2009 Apr;135(4):468-79.

2. McNamara JA Jr. A method of cephalometric evaluation. Am J Orthod. 1984 Dec;86(6):449-69.

3. Schwab RJ. Upper airway imaging. Clin Chest Med. 1998 Mar;19(1):33-54.

4. Abramson ZR, Susarla S, Tagoni JR, Kaban L. Three-dimensional computed tomographic analysis of airway anatomy. J Oral Maxillofac Surg. 2010 Feb;68(2):363-71.

5. Kim YJ, Hong JS, Hwang YI, Park YH. Three-dimensional analysis of pharyngeal airway in preadolescent children with accurate measurements with the true information on this region are prerequisites for correct diagnosis and treatment. ${ }^{23,24}$

Viewer software is necessary to visualize a CBCT scan, digital imaging and communications in medicine (DICOM). Among the commercially available viewers, Dolphin 3D is an excellent option that permits a variety of tridimensional analyses. ${ }^{11,25}$ However, many tools available in this program should be tested before they may be used clinically, as was done in this study. Furthermore, it is important to emphasize that although Dolphin 3D represents excellent technology, access is limited due to its high cost. ${ }^{26}$

The results show that the methodology proposed is reliable to evaluate upper airways, and may be used in further studies. In addition, the importance of the examiner's knowledge of the anatomy of this region cannot be underestimated, nor can the need for his/her specific training in the use of similar programs.

\section{Conclusion}

The values obtained in this study show an agreement between the two assessments of each observer and between the first evaluations of both observers for TV, NNA, and ONA, and allow reproducibility of the methodology. This tool can thus be recommended for use in future research studies, with the purpose of investigating the dimensions of upper airways, and, as such, contribute to the diagnosis of possible upper airway obstructions.

different anteroposterior skeletal patterns. Am J Orthod Dentofacial Orthop. 2010 Mar;137(3):306 e1-11; discussion 306-7.

6. Hatcher DC. Cone Beam computed tomography: craniofacial and airway analysis. Sleep Med Cli. 2010 Apr;5:59-70.

7. Iwasaki T, Hayasaki H, Takemoto Y, Kanomi R, Yamasaki Y. Oropharyngeal airway in children with Class III malocclusion evaluated by cone-beam computed tomography. Am J Orthod Dentofacial Orthop. 2009 Sep;136(3):318 e1-9; discussion 318-9.

8. Lenza MG, Lenza MM, Dalstra M, Melsen B, Cattaneo PM. An analysis of different approaches to the assessment of upper airway morphology: a CBCT study. Orthod Craniofac Res. 2010 May;13(2):96-105. 
9. Kikuchi Y. Three-dimensional relationship between pharyngeal airway and maxillo-facial morphology. Bull Tokyo Dent Coll. 2008 May;49(2):65-75.

10. Bland JM, Altman DG. Statistical methods for assessing agreement between two methods of clinical measurement. Lancet. 1986 Feb 8;1(8476):307-10.

11. El HP, Palomo JM. Measuring the airway in 3 dimensions: a reliability and accuracy study. Am J Orthod Dentofacial Orthop. 2010 Apr;137(4 Suppl):S50.e1-S.e9.

12. Grauer D, Cevidanes LS, Styner MA, Ackerman JL, Proffit WR. Pharyngeal airway volume and shape from cone-beam computed tomography: relationship to facial morphology. Am J Orthod Dentofacial Orthop. 2009 Dec;136(6):805-14.

13. Stratemann S, Huang JC, Maki K, Hatcher D, Miller AJ. Three-dimensional analysis of the airway with cone-beam computed tomography. Am J Orthod Dentofacial Orthop. 2011 Nov;140(5):607-15.

14. Pracharktam N, Nelson S, Hans MG, Broadbent BH, Redline $\mathrm{S}$, Rosenberg C, et al. Cephalometric assessment in obstructive sleep apnea. Am J Orthod Dentofacial Orthop. 1996 Apr;109(4):410-9.

15. Cevidanes L, Oliveira AE, Motta A, Phillips C, Burke B, Tyndall D. Head orientation in CBCT-generated cephalograms. Angle Orthod. 2009 Sep;79(5):971-7.

16. Oliveira AE, Cevidanes LH, Phillips C, Motta A, Burke B, Tyndall D. Observer reliability of three-dimensional cephalometric landmark identification on cone-beam computerized tomography. Oral Surg Oral Med Oral Pathol Oral Radiol Endod. 2009 Feb;107(2):256-65.

17. Shrout PE, Fleiss JL. Intraclass correlations: uses in assessing rater reliability. Psychol Bull. 1979 Mar;86(2):420-8.
18. Fleiss JL. Confidence intervals vs significance tests: quantitative interpretation. Am J Public Health. 1986 May;76(5):587-8.

19. Ludlow JB, Gubler M, Cevidanes L, Mol A. Precision of cephalometric landmark identification: cone-beam computed tomography vs conventional cephalometric views. Am J Orthod Dentofacial Orthop. 2009 Sep;136(3):312 e1-10; discussion -3.

20. Razavi T, Palmer R, Davies J, Wilson R, Palmer P. Accuracy of measuring the cortical bone thickness adjacent to dental implants using cone beam computed tomography. Clin Oral Implants Res. 2010 Jul;21(7):718-25.

21. Sun Z, Smith T, Kortam S, Kim DG, Tee BC, Fields H. Effect of bone thickness on alveolar bone-height measurements from cone-beam computed tomography images. Am J Orthod Dentofacial Orthop. 2011 Feb;139(2):e117-27.

22. Rankin G, Stokes M. Reliability of assessment tools in rehabilitation: an illustration of appropriate statistical analyses. Clin Rehabil. 1998 Jun;12(3):187-99.

23. Horner RL. Motor control of the pharyngeal musculature and implications for the pathogenesis of obstructive sleep apnea. Sleep. 1996 Dec;19(10):827-53.

24. Eisele HJ, Reichenberger F, Mayer K, Weissmann N, Seeger W, Schulz R. Obstructive sleep apnea in a patient with the Melkersson-Rosenthal syndrome. Sleep Breath. 2009 Sep;14(3):245-7.

25. German DS, German J. Cone-beam volumetric imaging: a twominute drill. J Clin Orthod. 2010 Apr;44(4):253-65; quiz 2.

26. Paixão MB, Sobral MC, Vogel CJ, Araujo TM. Comparative study between manual and digital cephalometric tracing using Dolphin Imaging software with lateral radiographs. Dental Press J Orthod 2010 Nov;15(6):123-30. 